\title{
Differential Expression of Tight Junctions and Cell Polarity Genes in Human Colon Cancer
}

\author{
Themistoklis Kourkoumpetis ${ }^{1,2}$, Kathryn E. Royse ${ }^{3}$, Liang Chen ${ }^{1,2,3}$, Milan Ravishankar ${ }^{1,2}$, \\ Michael Ittmann ${ }^{2,4}$, Hashem B. El-Serag ${ }^{1,2,3}$ and Li Jiao ${ }^{1,2,3^{*}}$
}

${ }^{1}$ Section of Gastroenterology and Hepatology, Baylor College of Medicine, Houston, USA; ${ }^{2}$ Michael E. DeBakey VA Medical Center, Houston, Texas, USA; ${ }^{3}$ Section of Health Service Research, Department of Medicine, Baylor College of Medicine, Houston, USA;

${ }^{4}$ Department of Pathology and Immunology, Baylor College of Medicine, Houston, USA

\begin{abstract}
Background and objectives: Tight junction and cell polarity proteins are paramount to epithelial cell polarity and transport. In vivo studies have shown the differential expression of tight junctions in colorectal cancer, with little to no human data to corroborate those findings. We investigated whether tight junction genes were differentially expressed in human colon cancer versus normal controls.
\end{abstract}

Methods: Total RNA was extracted from fresh frozen tumor tissues and normal adjacent tissues of 6 patients who were diagnosed with primary colon cancer as well as from normal mucosa of 5 unrelated polyp-free individuals. We used the Qiagen $\mathrm{RT}^{2}$ Profiler PCR array to determine the expression of 84 genes in the tight junction pathway. Student's $t$-test was used to compare gene expression levels between cancer tissues and normal mucosa using normalized gene expression data.

Results: Compared with normal mucosa, significant up-regulation of the claudin 1 (CLDN1) gene (fold-change = $16, p=0.001$ ) but down-regulation of the AMOTL1, CLDN5, JAM2 and TIAM1 genes (fold-change $>2, p<0.05$ ) were seen in colon cancer tissue.

Conclusions: We observed the differential expression of CLDN, AMOTL1, JAM2 and TIAM1 in colon cancer versus normal mucosa. Further larger studies are warranted to investigate the role of tight junction and cell polarity proteins in the progression of human colon tumors.

Introduction

The intestinal epithelium is a selective semipermeable barrier for luminal molecules and microbes. It maintains its complexity through

Keywords: Tight junction; Cell polarity; Gene expression; Colon cancer; Claudin. Abbreviations: AMOTL, angiomotin like; CGN, cingulin; CLDN, claudin; CTNNA, catenin alpha; JAM, junctional adhesion molecule; MPP, mitochondrial-processing peptidase; PARD, partitioning-defective protein; PCR, polymerase chain reaction; PTEN, phosphatase and tensin homolog; RNA, ribonucleic acid; RT, reverse transcription; SD, standard deviation; TIAM, t-cell lymphoma invasion and metastasis.

Received: October 18, 2017; Revised: January 23, 2018; Accepted: February 02, 2018

${ }^{*}$ Correspondence to: Li Jiao, Center for Innovations in Quality, Effectiveness and Safety (IQuESt), Michael E DeBakey VA Medical Center, 2002 Holcombe Blvd, MS152, Houston 77030, Texas, USA. Tel: 713-440-4456; Fax: 713-748-7359; Email: jiao@bcm.edu

How to cite this article: Kourkoumpetis T, Royse KE, Chen L, Ravishankar M, Ittmann M, El-Serag HB, Jiao L. Differential Expression of Tight Junctions and Cell Polarity Genes in Human Colon Cancer. Exploratory Research and Hypothesis in Medicine 2018;3(1):14-19. doi: 10.14218/ERHM.2017.00036. a diverse family of molecules that promote cell-to-cell anchorage and appropriate individual cell polarity, mainly being represented by the adherens junctions and tight junctions. ${ }^{1}$ Tight junctions are a diverse family of molecules which form an essential barrier between the internal and external environment and maintain its complexity.

Cell-to-cell adhesion is paramount to the maintenance of normal epithelial tissue architecture, and disruption of this anchorage is believed to aid to tumor progression and subsequent invasion to deeper tissues as well as distant metastasis. ${ }^{2}$ Intestinal barrier dysfunction is also an essential contributor to intestinal inflammation. ${ }^{3}$ Structural disruption of colonic epithelial cells might impact early tumorigenesis or progression to colorectal cancer. ${ }^{4}$ Changes in cell-to-cell junction molecules can lead to cellular migration, invasion and metastasis of cancer cells in a tissue-dependent manner. Tight junctions are protein complexes near the apical surface of the epithelial cell and have been found to promote cellular polarity, epithelial barrier maintenance and paracellular transport. ${ }^{1}$ Tight junctions are compiled by multiple building blocks, including claudins, which play a vital role in tight junction anchorage onto the cellular membrane. ${ }^{5}$ Claudins in- 
Table 1. Basic characteristics of cases with colon cancer and controls

\begin{tabular}{lll}
\hline Characteristic, mean (SD) or $\boldsymbol{n}(\%)$ & Cases, $\boldsymbol{n = 6}$ & Controls, $\boldsymbol{n}=\mathbf{5}$ \\
\hline Age in years & $61.3(5.3)$ & $62.4(9.1)$ \\
Sex, male & $6(100)$ & $5(100)$ \\
Race, Caucasian & $6(100)$ & $5(100)$ \\
Stage & & $\mathrm{NA}$ \\
$\quad 3(50)$ & $\mathrm{NA}$ \\
$\quad 3(50)$ & \\
$\quad$ Larly & & $2(40.0)$ \\
Colon segment & $2(33.3)$ & $3(60.0)$ \\
$\quad$ Sigmoid & $4(66.7)$ & \\
\hline
\end{tabular}

fluence cellular transformation to colon cancer as well as metastasis. ${ }^{6}$

Little is known about the role of tight junctions, claudin and polarity gene expression in human colon cancer development. In this study, we compared the expression levels of genes in the predefined tight junction pathway between colon cancer tissues and normal mucosa. We hypothesized that gene expression among the tight junction pathway is significantly altered in colon cancer compared to normal mucosa.

\section{Methods}

\section{Study participants and sample collection}

Our study consisted of 6 patients who were diagnosed with colon cancer and underwent total curative surgical excision at the affiliated hospitals of Baylor College of Medicine between 1999 and 2013. All study subjects had no personal history of malignancy and did not receive preoperative neoadjuvant chemotherapy. The research coordinators collected the tissue samples during the surgical procedure. The resected tumor tissue and normal adjacent mucosa samples were snap-frozen in liquid nitrogen and stored at $-80^{\circ} \mathrm{C}$ within 10 minutes from obtainment. The pathological report confirmed the diagnosis of colon adenocarcinoma, and the tumor volume for all was $>60 \%$. We defined the stage of cancer using the TNM definition of the American Joint Committee on Cancer. Early-stage colon cancer was defined by no regional lymph node metastasis and no distant metastasis.

In a colonoscopy-based case-control study, we also obtained colon mucosa samples from 5 unrelated individuals who underwent screening colonoscopy and were found to have a normal colon at the Michael E. DeBakey Veterans Affairs Medical Center, an affiliated hospital of Baylor College of Medicine. For each, the collected normal biopsy was immediately placed on the dry ice and transferred to $\mathrm{a}-80{ }^{\circ} \mathrm{C}$ freezer within 20 minutes. These samples served as the control tissues when the RNA quality of normal adjacent tissue of 5 cancer patients was not ideal for gene expression analysis. Each unrelated control was matched to each case per age ( \pm 5 years), sex, race/ethnicity, and colonic segment.

The patients provided written informed consent to allow their specimens for research before the surgery procedure.

\section{RNA extraction and gene expression analysis}

We extracted total RNA from fresh frozen tissue biopsies of 17 samples (6 biopsies from excised cancerous tissues, 6 biopsies from adjacent normal colon mucosa, and 5 from unrelated normal control mucosa) in the genomic core lab at the Texas Digestive Disease Center using the NucleoSpin RNA isolation kit (MACHEREY-NAGEL Inc, Bethlehem, PA, USA). We determined the nucleic acid 260:280 ratio to confirm the RNA purity of samples, which was $>2.0$ for all. However, the RNA integrity number was $<6.0$ for 5 normal adjacent mucosa samples. These RNA samples were deemed not adequate for gene expression analysis and thus were excluded from the gene expression experiment. As a result, the gene expression analysis was restricted to 6 resected colon cancers from 6 patients, 1 normal adjacent mucosa from 1 patient, and 5 normal mucosal biopsies from unrelated healthy controls.

We used the Qiagen RT ${ }^{2}$ Profiler PCR Array (Hilden, Germany) to determine the expression of 84 genes in the tight junction pathway. The array includes all claudin genes, and genes encoding OCLN, cell adhesion proteins (ESAM1, ICAM1, ICAM2 and PECAM1), junction adhesion proteins (F11R, IGSF5, JAM2 and alpha actinins), catenins (ACTN1-4, CTNNA1-3 and CTNNB1), junction interacting proteins (ACTN1-4, AMOTL1, GGN, YBX3, CTTN, EPB41, HCLS1, INADL, MAG1, MAG2, MLLT4, MPDZ, PARD3, SYMPK, TJAP1, TJP1-3, VAPA and ZAK), cytoskeleton regulation proteins (AMOT1, ASH1L, YBX3, CTTN, LLGL1, LLGL2, PARD3, PARD6A, PARD6B, SPTA1, SPTAN1, SPTB, SMURF1 and TIAM1), G protein signaling proteins (ARHGEF2, CDC42, CDK4, GNA1, RAC1 and RHOA), and protein kinases (CASK, CSNK2A1, CSNK2A2, CSNK2B, ILK, MAGI1, MAGI2, MARK2, MPP5, MPP6, PRKCI, PRKCZ and PTEN). The assays were conducted at the core facility at Baylor College of Medicine using the Bio-Rad LightCycler RealTime PCR System (Hercules, CA, USA). After the gene expression analysis, the data from 1 unrelated normal control showed poor signal and we eliminated the data from the analysis. Therefore, we included 6 colon cancer tissue and 5 control tissues in this analysis.

The institutional review board of Baylor College of Medicine and Michael E. DeBakey Veterans Affairs Medical Center approved the study protocol.

\section{Statistical analysis}

We used the RT ${ }^{2}$ Profiler PCR Array Data Analysis Software, version 3.5, for data analysis. Student's $t$-test was used to compare the expression levels between cancer and normal mucosa using normalized gene expression data against $A C T B, H P R T 1$ and $R P L P 0$ as the housekeeping genes. We also exploratorily conducted the 
A

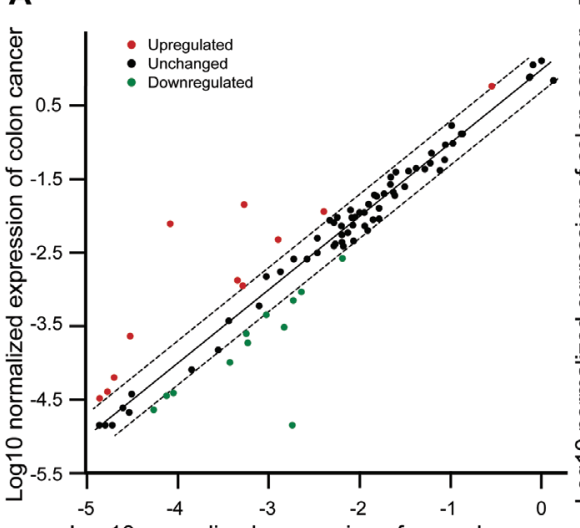

Log10 normalized expression of normal mucosa
B

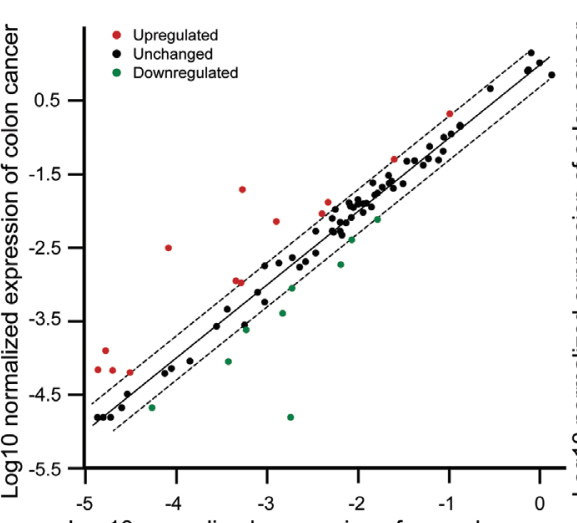

Log10 normalized expression of normal mucosa
C

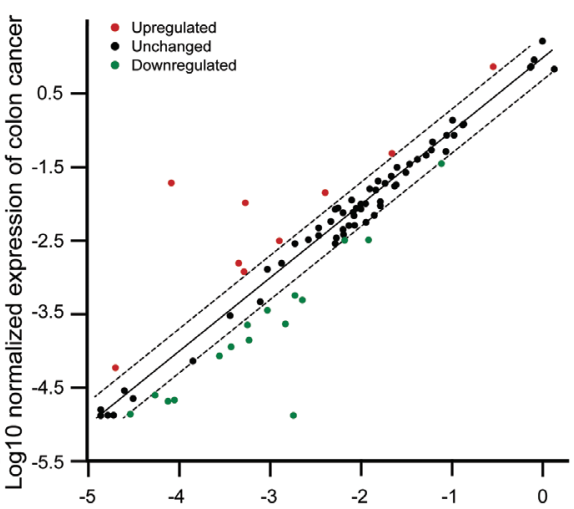

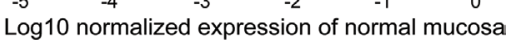

Fig. 1. Comparison of gene expression between all colon cancer and normal mucosa (A), between early-stage colon cancer and normal mucosa (B) and between late-stage colon cancer and normal mucosa (C). Scatter plot was used to compare the normalized expression of every gene on the array between two groups by plotting them against each other. The central lines indicate the constant gene expression. The dotted lines indicate the selected fold-regulation threshold. Red dots, up-regulated; black dots, unchanged; green dots, down-regulated. The figure was generated from the RT ${ }^{2}$ Profiler PCR Array Data Analysis Software, version 3.5.

analysis by cancer stage, i.e. early-stage cancer or late-stage cancer. We calculated fold-change/fold-regulation using the ${ }^{\Delta \Delta} \mathrm{Ct}$ method, in which delta $\mathrm{Ct}$ was calculated between the gene of interest and an average of reference housekeeping genes, followed by the ${ }^{\Delta \Delta} \mathrm{Ct}$ calculation: ${ }^{\Delta} \mathrm{Ct}$ (cancer group) ${ }^{\Delta} \mathrm{Ct}$ (control group). Fold-change was then calculated using the $2^{\wedge}\left({ }^{\Delta \Delta} \mathrm{Ct}\right)$ formula.

The scatter plot compares the normalized expression of every gene on the array between the two groups by plotting them against one another to visualize significant gene expression changes. The central line indicates unchanged gene expression. The dotted lines indicate the selected fold-regulation threshold. Data points beyond the dotted lines in the upper left and lower right sections meet the selected fold-regulation threshold. Multiple testing for 84 genes was adjusted using false discovery rate. Raw $p$-value of $<0.01$ was indicative of statistical significance in this study.

Gene Set Enrichment Analysis was performed using WebGestalt (http://www.webgestalt.org) for Kyoto Encyclopedia of Genes and Genomes pathway analysis to determine the enrichment scores and functional significance of all genes in the panel follow- ing $\log 2$ conversion of their fold-changes. ${ }^{7}$

\section{Results}

The 6 cancer patients were all non-Hispanic Caucasian men. The age range was 55 to 75 years. Three patients had an early-stage tumor and three patients had a late-stage tumor (Table 1).

When colon cancer was compared with healthy controls, the differential expression of multiple genes was observed (Fig. 1). The $C L D N 1$ gene was significantly up-regulated, with fold-change of $16(p<0.001)$ (Table 2). Four genes were down-regulated after multiple testing adjustments, including AMOTL1, CLDN5, JAM2 and TIAM1. The differential expression was persistently seen in early-stage as well as late-stage tumors. The down-regulation of CTNNA3, JAM3, MPP5 and PTEN was not statistically significant after adjustment for multiple testing. CLDN2 was up-regulated by $>25$-fold and CLDN8 was down-regulated $>100$-fold in tumor tissue compared with control mucosa. However, the difference was

Table 2. Differential expression of genes in human colon cancer tissue and normal control tissue

\begin{tabular}{llllllc}
\hline Gene & $\begin{array}{l}\text { All cases versus } \\
\text { controls, fold-change }\end{array}$ & Crude $\boldsymbol{P}$ & $\begin{array}{l}\text { Early stage cancer versus } \\
\text { controls, fold-change }\end{array}$ & Crude $\boldsymbol{P}$ & $\begin{array}{l}\text { Late stage cancer versus } \\
\text { controls, fold-change }\end{array}$ & $\begin{array}{l}\text { Crude } \boldsymbol{P} \\
\text { AMOTL1 }\end{array}$ \\
\hline CLDN1 & -3.13 & 0.007 & -2.74 & 0.07 & -3.57 & 0.03 \\
CLDN5 & -4.62 & 0.008 & 23.14 & $<0.0001$ & 11.94 & 0.10 \\
CTNNA3 & -3.24 & 0.004 & -3.63 & 0.05 & -6.60 & 0.03 \\
JAM2 & -7.18 & 0.010 & -3.45 & 0.03 & -3.04 & 0.09 \\
JAM3 & -2.94 & 0.002 & -5.36 & 0.03 & -9.60 & 0.02 \\
MPP5 & -2.18 & 0.012 & -2.29 & 0.11 & -3.77 & 0.04 \\
PARD6A & 1.67 & 0.015 & -2.43 & 0.08 & -1.96 & 0.08 \\
PTEN & -2.36 & 0.092 & 2.54 & 0.004 & 1.09 & 0.64 \\
TIAM1 & -4.77 & 0.013 & -2.75 & 0.07 & -2.02 & 0.08 \\
CGN & -2.09 & 0.008 & -5.27 & 0.06 & -4.32 & 0.04 \\
\hline
\end{tabular}




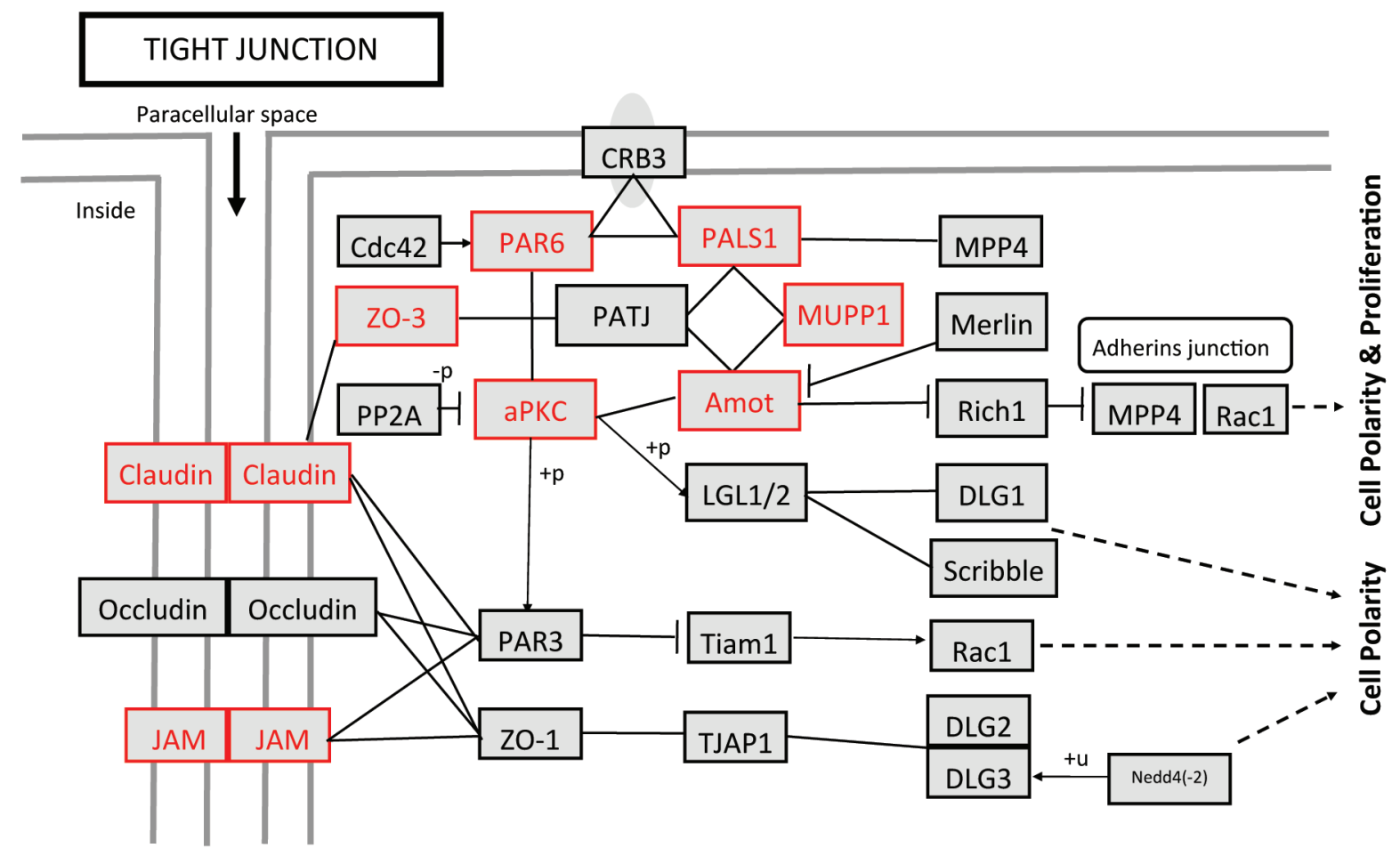

*Significantly enriched in KEGG Pathway

Fig. 2. Pathway identified by gene set enrichment analysis and Kyoto Encyclopedia of Genes and Genomes pathway analysis. Red indicates the significantly enriched genes.

not statistically significant (unadjusted $p>0.05$ ). Overall, gene signatures and pathway analysis showed down-regulation of tight junction genes in colon cancer compared with normal mucosa (Supplemental Table S1).

We identified three significantly positively enriched Kyoto Encyclopedia of Genes and Genomes pathways (false discovery rate $p<0.001$ ), including cell adhesion molecules, leukocyte transendothelial migration and tight junction. Significant contributors to all three sets were members of the claudin family. The primary differentially enriched genes of the tight junction pathway were those related to cell polarity and proliferation and junction assembly (Fig. 2).

\section{Discussion}

Our pilot findings suggested an altered expression of multiple tight junctions and cell polarity genes in colon cancer compared with normal colonic tissues. Specifically, AMOTL1, CLDN5, JAM2 and TIAM1 were the genes significantly down-regulated and CLDN1 was the only gene significantly up-regulated in colon cancer compared with normal mucosa.

Claudins are a diverse family of transmembrane proteins that aid in the adhesion of tight junction complexes to the intracellular cytoskeleton. ${ }^{8}$ At least 24 subtypes have been described, with some of them having a specific tissue predominance and differential expression in specific malignancies. ${ }^{8}$ In our study, CLDN1 was up-regulated in colon cancer compared with normal mucosa. It has been proposed that increased CLDN1 expression could pro- mote malignant cell behavior via a decrease in E-cadherin by increasing expression of ZEB-1, beta-catenin nuclear translocation and WNT signaling. ${ }^{6}$ Our findings were consistent with previous findings in premalignant conditions in humans. Weber et al. ${ }^{9}$ have shown $C L D N 1$ to be significantly up-regulated in active inflammatory bowel disease, colorectal adenomas and inflammatory bowel disease-associated dysplasia. Bujko et al. ${ }^{5}$ found increased CLDN1 expression in 26 samples of colorectal cancer, and 42 adenomas compared to normal colon tissue. In contrast, Ersoz et al. ${ }^{2}$ and Suren et al. ${ }^{10}$ have shown that $C L D N 1$ expression was significantly down-regulated in a series of colon cancer tissues and this lower expression was linked to a higher proportion of lymph node metastasis or aggressive behavior of colorectal carcinoma. ${ }^{2,10}$ This difference might be explained by the different gene expression approach used. We evaluated the gene expression at the mRNA levels, whereas Suren et al. ${ }^{10}$ and Matsuoka et al..$^{11}$ used immunohistochemical testing.

We found CLDN5 was significantly down-regulated and $C L D N 2$ was insignificantly up-regulated in cancer tissues compared with control mucosa. CLDN2 is a pore-forming protein and CLDN5 is a seal-forming protein, and increased expression of CLDN2 and decreased expression of CLDN5 are consistent with epithelial dysfunction, as characterized by reduced barrier resistance. ${ }^{2,5,12} C L D N 5$ was only shown to be down-regulated in one human study and its role in colorectal tumorigenesis has not been well elucidated. ${ }^{2}$ We did not find significant differential expression of other $C L D N$ genes between cancer and normal mucosa, although $C L D N 6,7$ and 8 were all non-significantly down-regulated in colon cancer tissue. Nakayama et al. ${ }^{12}$ have shown that $C L D N 7$ expression was significantly down-regulated in human 
colon cancer cell lines through hypermethylation of the CLDN7 promoter region. Gene Set Enrichment Analysis revealed a statistically significant difference in the enrichment of these $C L D N$ genes in the Kyoto Encyclopedia of Genes and Genomes tight junction, cell adhesion molecules, and leukocyte transendothelial migration pathways. Further clinical studies are warranted to examine the implication of claudins in colorectal cancer development.

Junctional adhesion molecule proteins are part of the immunoglobulin subfamily and involved in the formation of tight junctions in both endothelial and epithelial cells. ${ }^{13}$ These transmembrane proteins are believed to aid in cell polarity by anchoring adaptor proteins and tight junctions. ${ }^{13,14}$ We found JAM2 to be down-regulated in colon tumor tissue. Down-regulation of JAM2 has also been observed in two previous studies. ${ }^{5,15}$ Colon cancer cells transfected to overexpress $J A M 2$ were found to have significantly reduced growth, invasion and migration relative to cancer cells with low expression. ${ }^{14}$ In contrast, JAM deficiency had a protective effect against melanoma lung metastasis in mice. ${ }^{14}$ This is likely due to the role of JAM2 in cell adhesion to endothelium, preventing transendothelial transmigration. ${ }^{14}$ In the Gene Set Enrichment Analysis, the leukocyte transendothelial migration pathway was found to be significantly enriched. Analysis of integrated epigenome and transcriptome data identified the epigenetic modulation of cell adhesion molecules in colorectal tumor, with JAM2 being one of four genes down-regulated by hypermethylation. All these studies pinpoint the importance of JAM2 in colorectal carcinogenesis. ${ }^{16}$

The TIAMI gene encodes a protein that aids in cell anchoring. ${ }^{17}$ In contrast to our study that showed TIAM1 was significantly down-regulated in colon cancer tissues, TIAMI has been shown to be over-expressed in many types of cancers, such as breast and esophageal adenocarcinomas. ${ }^{17,18}$ In oral squamous cell carcinoma, the increased expression of TIAM1 was correlated with lymph node metastasis, earlier recurrence and worse prognosis. ${ }^{19}$ One in vivo study found that TIAMI promotes invasion and metastasis of colorectal cancer, possibly through activation of the Wnt/betacatenin signaling pathway in a TIAM1 transgenic mouse model. ${ }^{20}$ In line with our observation, it has also been shown that suppression of TIAM1 is required for enhanced cell migration and invasion in human colorectal cancer enteroid models. ${ }^{21}$

Our findings also show significant down-regulation of AMOTL1 in colon cancer tissues compared with normal mucosa. This gene controls endothelial cell migration and polarity aiding in cancerous angiogenesis, and its down-regulation has been shown in studies involving cervical cancer and breast cancer. ${ }^{22,23}$ To our knowledge, this is the first evidence that AMOTL1 was down-regulated in colon cancer tissues compared with normal mucosa.

Our study provides novel evidence on the significant differential expression of tight junction genes in human colon cancer compared with normal mucosa. However, several limitations should be noted. First, this was a preliminary retrospective study with small sample size. Thus, false positive and false negative findings could have occurred. The small sample size prohibited the potential for valid subgroup analyses. We also did not collect complete clinical and survival data from our patients and the survival data were not documented for research purposes. This study will be followed by an investigation on the role of tight junction genes on survival of patients with colon cancer in the context of comprehensive clinical metadata and known risk factors for colorectal cancer.

\section{Future research directions}

We will conduct a large clinical study collecting more comprehen- sive clinical data on survival of patients with colorectal cancer. If tight junction and cell polarity mechanisms are shown to be causally associated with colorectal cancer survival, dysregulation of tight junctions can be used as potential markers for the prognosis of colorectal cancer. Future studies should also identify microbiome or diet or targeted therapeutic agents to modulate tight junction and cell polarity (such as blocking claudin 1) in pre-malignant or malignant lesions.

\section{Conclusions}

In conclusion, in colon cancer, the expression of multiple genes related to tight junctions and cell polarity was found to be significantly altered compared with controls. Understanding the molecular mechanism of claudins and tight junction disruption and its clinical significance in epithelial to mesenchymal transition may provide novel insight into colorectal carcinogenesis. ${ }^{24}$

\section{Acknowledgments}

This research was supported by the Gillson Longenbaugh Foundation and Golfers Against Cancer organization (PI: LJ), Cancer Prevention Research Institute of Texas (RP\#140767, PI: LJ). National Cancer Institute P30 Cancer Center (P30 CA125123) for support of the Human Tissue Acquisition and Pathology Shared Resources. This project was supported in part by PHS grant P30DK056338 and the expert assistance of Lisa D. White, Ph.D. The research was also supported in part by the Houston VA HSR\&D Center for Innovations in Quality, Effectiveness and Safety (CIN13-413).

\section{Conflict of interest}

The authors have no conflict of interests related to this publication.

\section{Author contributions}

Study design (LJ), sample acquisition (MI, HBE), data analysis (KER, LC), data interpretation and manuscript writing (TK, KER, MR), critical intellectual input (TK, KER, LC, MR, MI, HBE, LJ).

\section{Supporting information}

Supplementary material for this article is available at https://doi. org/10.14218/ERHM.2017.00036.

Supplemental Table S1. Differential expression of genes in human colon cancer tissue $(n=6)$ and normal control tissues $(n=5)$.

\section{References}

[1] Groschwitz KR, Hogan SP. Intestinal barrier function: molecular regulation and disease pathogenesis. J Allergy Clin Immunol 2009;124:320. doi:10.1016/j.jaci.2009.05.038.

[2] Ersoz S, Mungan S, Cobanoglu U, Turgutalp H, Ozoran Y. Prognostic importance of Claudin-1 and Claudin-4 expression in colon carcinomas. Pathol Res Pract 2011;207:285-289. doi:10.1016/j.prp.2011.01. 011. 
[3] Wyatt J, Vogelsang H, Hübl W, Waldhöer T, Lochs H. Intestinal permeability and the prediction of relapse in Crohn's disease. Lancet 1993;341:1437-1439. doi:10.1016/0140-6736(93)90882-H.

[4] Soler AP, Miller RD, Laughlin KV, Carp NZ, Klurfeld DM, Mullin JM. Increased tight junctional permeability is associated with the development of colon cancer. Carcinogenesis 1999;20(8):1425-1431. doi:10.1093/carcin/20.8.1425.

[5] Bujko M, Kober P, Mikula M, Ligaj M, Ostrowski J, Siedlecki JA. Expression changes of cell-cell adhesion-related genes in colorectal tumors. Oncol Lett 2015;9(6):2463-2470. doi:10.3892/ol.2015.3107.

[6] Dhawan P, Singh AB, Deane NG, No Y, Shiou SR, Schmidt C, et al. Claudin-1 regulates cellular transformation and metastatic behavior in colon cancer. J Clin Invest 2005;115(7):1765-1776. doi:10.1172/ $\mathrm{JCl} 24543$.

[7] Wang J, Vasaikar S, Shi Z, Greer M, Zhang B. WebGestalt 2017: a more comprehensive, powerful, flexible and interactive gene set enrichment analysis toolkit. Nucleic Acids Res 2017;45(W1):W130-W137. doi:10.1093/nar/gkx356.

[8] Shin K, Fogg VC, Margolis B. Tight junctions and cell polarity. Annu Rev Cell Dev Biol 2006;22:207-235. doi:10.1146/annurev.cellbio.22.010305.104219.

[9] Weber CR, Nalle SC, Tretiakova M, Rubin DT, Turner JR. Claudin-1 and claudin-2 expression is elevated in inflammatory bowel disease and may contribute to early neoplastic transformation. Lab Invest 2008;88(10):1110-1120. doi:10.1038/labinvest.2008.78.

[10] Suren D, Yildirim M, Kaya V, Alikanoglu AS, Bulbuller N, Yildiz M, et al. Loss of tight junction proteins (Claudin 1, 4, and 7) correlates with aggressive behavior in colorectal carcinoma. Med Sci Monit 2014;20:1255-1262. doi:10.12659/MSM.890598.

[11] Matsuoka T, Mitomi H, Fukui N, Kanazawa H, Saito T, Hayashi T, et al. Cluster analysis of claudin-1 and -4, E-cadherin, and beta-catenin expression in colorectal cancers. J Surg Oncol 2011;103(7):674-686. doi:10.1002/jso.21854

[12] Nakayama F, Semba S, Usami Y, Chiba H, Sawada N, Yokozaki H. Hypermethylation-modulated downregulation of claudin-7 expression promotes the progression of colorectal carcinoma. Pathobiology 2008;75(3):177-185. doi:10.1159/000124978.

[13] Zhao $H$, Yu H, Martin TA, Teng $X$, Jiang WG. The role of JAM-B in cancer and cancer metastasis (Review). Oncol Rep 2016;36(1):3-9. doi:10.3892/or.2016.4773.
[14] Meguenani M, Miljkovic-Licina M, Fagiani E, Ropraz P, Hammel $P$, Aurrand-Lions $\mathrm{M}$, et al. Junctional adhesion molecule $\mathrm{B}$ interferes with angiogenic VEGF/VEGFR2 signaling. FASEB J 2015;29:34113425. doi:10.1096/fj.15-270223.

[15] Zhao H, Yu H, Martin TA, Zhang Y, Chen G, Jiang WG. Effect of junctional adhesion molecule-2 expression on cell growth, invasion and migration in human colorectal cancer. Int J Oncol 2016;48(3):929936. doi:10.3892/ijo.2016.3340.

[16] Kok-Sin T, Mokhtar NM, Ali Hassan NZ, Sagap I, Mohamed Rose I, Harun $\mathrm{R}$, et al. Identification of diagnostic markers in colorectal cancer via integrative epigenomics and genomics data. Oncol Rep 2015;34(1):22-32. doi:10.3892/or.2015.3993.

[17] Liu H, Shi G, Liu X, Wu H, Fan Q, Wang X. Overexpression of Tiam1 predicts poor prognosis in patients with esophageal squamous cell carcinoma. Oncol Rep 2011;25:841-848. doi:10.3892/or.2010.1122.

[18] Jin H, Li T, Ding Y, Deng Y, Zhang W, Yang H, et al. Methylation status of T-lymphoma invasion and metastasis 1 promoter and its overexpression in colorectal cancer. Hum Pathol 2011;42:541-551. doi:10.1016/j.humpath.2010.08.013.

[19] Lin L, You J, Qian Y, Han Y, Xiong H, Zhu T, et al. The prognostic value of T Lymphoma Invasion and Metastasis 1 (TIAM1) expression in oral squamous cell carcinoma. J Biochem Mol Toxicol 2017;31(4):e21875. doi:10.1002/jbt.21875.

[20] Yu LN, Zhang QL, Li X, Hua X, Cui YM, Zhang NJ, et al. Tiam1 transgenic mice display increased tumor invasive and metastatic potential of colorectal cancer after 1,2-dimethylhydrazine treatment. PLoS One 2013;8:e73077. doi:10.1371/journal.pone.0073077.

21] Cottonham $\mathrm{CL}$, Kaneko $\mathrm{S}, \mathrm{Xu}$ L. miR-21 and miR-31 converge on TIAM1 to regulate migration and invasion of colon carcinoma cells. J Biol Chem 2010;285:35293-35302. doi:10.1074/jbc.M110.160069.

[22] Wan HY, Li QQ, Zhang Y, Tian W, Li YN, Liu M, et al. MiR-124 represses vasculogenic mimicry and cell motility by targeting amotL1 in cervical cancer cells. Cancer Lett 2014;355(1):148-158. doi:10.1016/j. canlet.2014.09.005.

[23] Couderc C, Boin A, Fuhrmann L, Vincent-Salomon A, Mandati V, Kieffer $\mathrm{Y}$, et al. AMOTL1 Promotes Breast Cancer Progression and Is Antagonized by Merlin. Neoplasia 2016;18(1):10-24. doi:10.1016/j. neo.2015.11.010.

[24] Kwon MJ. Emerging roles of claudins in human cancer. Int J Mol Sci 2013;14(9):18148-18180. doi:10.3390/ijms140918148. 